\title{
Use of administrative claims data for comparative effectiveness research of rheumatoid arthritis treatments
}

\author{
Seo Young Kim ${ }^{1,2 *}$ and Daniel H Solomon ${ }^{1,2}$ \\ See related research by Curtis et al., http://arthritis-research.com/content/13/5/R155
}

\begin{abstract}
Observational studies, particularly those using large administrative claims databases, have become increasingly popular sources of comparative effectiveness or comparative safety research. Studies using claims data often face challenges and criticisms due to the lack of certain clinical information, such as lifestyle risk factors, disease severity, and questionable accuracy of disease diagnoses. A novel, claims-based algorithm to evaluate the clinical effectiveness of rheumatoid arthritis medications has been developed and its performance seems promising, although further validation is needed.
\end{abstract}

Since the first approval of biologic therapy for rheumatoid arthritis (RA) in 1998, the treatment of the disease has changed substantially. A number of different biologic agents targeting various cytokines are currently available, but few data exist comparing the effectiveness of one biologic agent with another, highlighting the importance and need for comparative effectiveness research (CER) in RA [1]. In fact, comparative effectiveness of biologic therapy in RA was one of the top 25 priority research topics recommended by the Institute of Medicine in 2009 [2].

A number of different study designs such as randomized clinical trials, meta-analyses of randomized clinical trials, and observational studies using various data sources including patient registries, electronic medical records, and administrative claims databases can be considered for CER. The use of randomized clinical trials

*Correspondence: skim62@partners.org

'Division of Rheumatology, Immunology and Allergy, Brigham and Women's

Hospital, 1620 Tremont Street, Suite 3030, Boston MA 02120, USA

Full list of author information is available at the end of the article is limited in CER because of intrinsic weaknesses such as lack of generalizability, insufficient sample size, inadequate follow-up time, and high cost. Observational study designs include prospective registries and retrospective analysis of administrative healthcare data, often collected for insurance payment.

Prospective RA patient registries have a number of benefits, including detailed information on RA diagnosis, disease activity, and treatment, but often have limited generalizability and sample size, and incomplete data on comorbidities and other medications [3]. Observational studies, particularly those using large administrative claims databases, have therefore become increasingly popular sources of CER or comparative safety research, because they have several important strengths such as large size and efficiency, generalizability, high validity and completeness of prescription drug data, and low cost [4]. Furthermore, a previous validation study showed that RA patients can be accurately identified using a combination of diagnosis codes and disease-modifying antirheumatic drug (DMARD) prescriptions in claims data [5]. However, pharmacoepidemiologic studies using claims data face challenges and criticisms due to the lack of certain clinical information, such as lifestyle risk factors, disease severity, and questionable accuracy of disease diagnoses. A number of previous studies successfully used claims data to assess comparative effectiveness of DMARDs on specific outcomes [6,7], but not so much research has been done to compare the effectiveness of DMARDs in RA activity.

In the current issue of Arthritis Research \& Therapy, Curtis and colleagues present the development and validation of a novel, claims-based algorithm to evaluate the clinical effectiveness of RA medications [1]. This study has an important implication in CER of RA and shows the potential for using the claims data to compare the clinical effectiveness of multiple biologic or nonbiologic DMARDs in large real-world populations. RA patients initiating one of the biologic agents (abatacept, 
adalimumab, etanercept, infliximab and rituximab) were identified based on the data from the longitudinal Veterans Affairs RA registry linked to the Veterans Health Administration medical and pharmacy claims [1]. The effectiveness algorithm consists of strict, a priori defined criteria: high drug adherence, an increase in biologic dose compared with the starting dose, switching to a different biologic or adding a new nonbiologic DMARD, initiation of chronic glucocorticoids, an increase in glucocorticoid dose during the follow-up period, and more than one parenteral or intra-articular injection on a given day after the patient had been on biologic treatment for longer than 3 months. The gold standard for effectiveness was defined as 28-joint Disease Activity Score <3.2 (low disease activity) or improvement in 28-joint Disease Activity Score $>1.2$ units at the 1-year follow-up visit following the index visit. In the authors' preliminary assessment, the algorithm seems promising with good performance characteristics, ranging from 75 to $90 \%$ [1].

While this study represents an important effort, several potential pitfalls in this claims-based effectiveness algorithm should be noted. First, performance of the algorithm may be database dependent. In other words, the algorithm may perform differently in a commercially insured or Medicare population versus the Veterans Affairs population in which it was developed. Whether the algorithm will have a similar performance in other claims databases therefore needs to be further examined. Second, as the algorithm required patients to have high adherence to DMARDs (over 80\%), it may not perform well in nonadherent patients. One cannot therefore assume the algorithm represents good disease control since it was developed in a population who were medication adherent. Third, the performance of the effectiveness algorithm was assessed at 1-year follow-up. As the authors suggested, the validity of the algorithm should be confirmed at different time points.

A claims-based effectiveness algorithm with acceptable performance characteristics across different data settings will be a powerful and desired tool for CER of RA. Such an algorithm will enable large-scale, population-based studies comparing the effectiveness of different DMARD regimens. Such studies will facilitate head-to-head comparisons, supplementing typical randomized controlled trials and prospective registries that usually include disease activity.

\section{Abbreviations}

CER, comparative effectiveness research; DMARD, disease-modifying antirheumatic drug; RA, rheumatoid arthritis.

\section{Competing interests}

SYK has received research support from Takeda Pharmaceuticals North America and Pfizer. DHS has received research support from Abbott Immunology, Amgen, Lilly, and an educational grant from Bristol-Myers Squibb. He serves as an unpaid member of an Executive Committee and a Data Safety Monitoring Board for two analgesic trials sponsored by Pfizer.

\section{Acknowledgements}

SYK is supported by the NIH grant K23 AR059677. DHS is supported by the NIH grants K24 AR055989, P60 AR047782, R21 DE018750, and R01 AR056215.

\section{Author details}

'Division of Rheumatology, Immunology and Allergy, Brigham and Women's Hospital, 1620 Tremont Street, Suite 3030, Boston MA 02120, USA. ²Division of Pharmacoepidemiology and Pharmacoeconomics, Brigham and Women's Hospital, 1620 Tremont Street, Suite 3030, Boston MA 02120, USA.

Published: 7 October 2011

\section{References}

1. Curtis JR, Baddley JW, Yang S, Patkar N, Chen L, Delzell E, Mikuls TR, Saag KG, Singh J, Safford M, Cannon GW: Derivation and preliminary validation of an administrative claims-based algorithm for the effectiveness of medications for rheumatoid arthritis. Arthritis Res Ther 2011, 13:R155.

2. Institute of Medicine: Initial National Priorities for Comparative Effectiveness Research. Washington, DC: Institute of Medicine; 2009.

3. Curtis JR, Jain A, Askling J, Bridges SL Jr, Carmona L, Dixon W, Finckh A, Hyrich K, Greenberg JD, Kremer J, Listing J, Michaud K, Mikuls T, Shadick N, Solomon $\mathrm{DH}$, Weinblatt ME, Wolfe F, Zink A: A comparison of patient characteristics and outcomes in selected European and U.S. rheumatoid arthritis registries. Semin Arthritis Rheum 2010, 40:2-14.

4. Schneeweiss $S$, Avorn J: A review of uses of health care utilization databases for epidemiologic research on therapeutics. J Clin Epidemiol 2005, 58:323-327.

5. Kim S, Servi A, Polinski J, Mogun H, Weinblatt M, Katz J, Solomon DH: Validation of rheumatoid arthritis diagnoses in health care utilization data. Arthritis Res Ther 2011, 13:R32.

6. Solomon DH, Avorn J, Katz JN, Weinblatt ME, Setoguchi S, Levin R, Schneeweiss S: Immunosuppressive medications and hospitalization for cardiovascular events in patients with rheumatoid arthritis. Arthritis Rheum 2006, 54:3790-3798.

7. Solomon D, Massarotti E, Garg R, Liu J, Canning C, Schneeweiss S: Association between disease-modifying antirheumatic drugs and diabetes risk in patients with rheumatoid arthritis and psoriasis. JAMA 2011, 305:2525-2531.

doi:10.1186/ar3472

Cite this article as: Kim SY, Solomon DH: Use of administrative claims data for comparative effectiveness research of rheumatoid arthritis treatments. Arthritis Research \& Therapy 2011, 13:129. 